\title{
A FRET-Based Approach for Quantitative Evaluation of Forskolin-Induced Pendrin Trafficking at the Plasma Membrane in Bronchial NCI H292 Cells
}

\author{
Grazia Tamma Marianna Ranieria Silvia Dossena ${ }^{\mathrm{b}} \quad$ Annarita Di Mise ${ }^{\mathrm{a}}$ \\ Charity Nofziger $^{b} \quad$ Maria Svelto ${ }^{a, c}$ Markus Paulmichl ${ }^{b}$ Giovanna Valentia,c \\ aDept. of Biosciences Biotechnologies and Biopharmaceutics, University of Bari, Italy; ' $I n s t i t u t e ~ o f$ \\ Pharmacology and Toxicology, Paracelsus Medical University, Salzburg, Austria; ' ${ }^{\circ}$ entre of Excellence \\ Genomic and Proteomics GEBCA, University of Bari, Italy
}

\section{Key Words}

Pendrin • Forskolin • Actin • Trafficking • FRET

\begin{abstract}
Background: Human pendrin (SLC26A4, PDS) is an integral membrane protein acting as an electroneutral anion exchanger. Loss of function mutations in pendrin protein cause Pendred syndrome, a disorder characterized by sensorineural deafness and a partial iodide organification defect that may lead to thyroid goiter. Additionally, pendrin up-regulation could play a role in the pathogenesis of several diseases including bronchial asthma and chronic obstructive pulmonary disease (COPD). Therefore, monitoring the plasma membrane abundance and trafficking of pendrin in the context of a living cell is crucially important. Methods: Trafficking of pendrin to the plasma membrane was monitored by fluorescence resonance energy transfer (FRET), a physical phenomenon occurring between two fluorophores (the FRET donor and acceptor) located in close spatial proximity. Because the efficiency of the energy transfer is inversely proportional to the sixth power of the distance between donor and acceptor, FRET is extremely sensitive to small changes in distance between the donor and acceptor and is therefore a powerful tool to determine protein-protein interactions. Results: FRET studies revealed that forskolin-induced CAMP production is associated with a significant increase of pendrin expression at plasma membrane, which is paralleled by a decrease in intracellular $\mathrm{pH}$. Pendrin transposition to the membrane is accompanied with a partial depolymerization of actin cytoskeleton via Rho-GTPase inhibition. Conclusion: Trafficking to the plasma membrane is critical in the regulation of pendrin activity. Therefore, reliable tools for monitoring and quantifying this phenomenon are highly desirable.
\end{abstract}




\section{Introduction}

Human pendrin (SLC26A4, PDS) is a 780 amino acid membrane protein with 12 putative transmembrane domains with transport features distinct from those of the other members of the SLC26 family, since it acts as a sodium-independent anion exchanger, exclusively for monovalent anions including iodide, chloride, bicarbonate, hydroxide and thiocyanate [1-3]. These biophysical properties are fundamental to the multiple physiological functions of the transporter and its involvement in many pathological processes. In line with this, pendrin is expressed in different tissues and organs such as the thyroid, kidney, inner ear, mammary gland, testis, placenta, endometrium, lung, ameloblasts and liver [4-7]. Loss of function mutations in pendrin protein cause Pendred syndrome, an autosomal recessive inherited disease characterized by severe bilateral hearing loss, inner ear anomalies and a partial iodide organification defect that may lead to the development of euthyroid goiter or hypothyroidism [8-11]. Pendrin up/dys-regulation, may indeed contribute to several human diseases. For example, in the kidney, pendrin might play a role in the pathogenesis of hypertension, while overexpression of the transporter in the lung may exacerbate respiratory disorders such as bronchial asthma and chronic obstructive pulmonary disease (COPD). Altered pendrin gene expression may be part of signal transduction pathways characterizing the onset of specific human diseases. Development of bronchial asthma and COPD is in part dependent on signaling mediated by interleukin-4/interleukin-13 (IL-4/IL-13), which modulates the expression level of several genes including pendrin, via STAT-6 [12].

To gain an integrated understanding of the physiological role of proteins, it is critically important to develop advanced methods for monitoring not only gene expression but also the sub-cellular localization of proteins in the context of a living cell. It is well accepted that the abundance of pendrin at the plasma membrane is a strictly regulated process. In the thyroid, the abundance of pendrin at the plasma membrane level is controlled by cAMP/ PKA signaling $[13,14]$. In the kidney, the role of cAMP/PKA signaling in the regulation of pendrin expression and trafficking is controversial. Azroyan et al. found that short-term (20 min) stimulation of the cAMP/PKA pathway increased the amount of pendrin at the cell surface as well as its transport activity in stably transfected opossum kidney proximal cells [15]. Others found that long-term (overnight) elevation of cAMP increased pendrin total protein abundance in both cultured mouse cortical collecting duct and connecting tubule, while short-term (30 min) treatment with forskolin (an adenylyl cyclase agonist) did not alter pendrin protein abundance or its subcellular distribution [16]. In the lung, cAMP increased the volume and $\mathrm{pH}$ of the secreted fluid via stimulation of CFTR and pendrin activity, respectively [17]. Together these findings underscore the tight relation linking gene expression and regulated intracellular trafficking with pendrin function.

In this study, we quantitatively assessed the abundance of pendrin at the plasma membrane in living cells using fluorescence resonance energy transfer (FRET). FRET is a physical process by which the energy of an excited fluorophore (the FRET donor) is transferred to a nearby fluorescent molecule (the FRET acceptor). The "conditio sine qua non" for energy transfer is the close spatial proximity of the donor and acceptor, which must be within the range of $1-10 \mathrm{~nm}$. This important feature makes FRET a powerful tool to evaluate protein-protein interactions with spatial and temporal resolution [18] in fixed and living cells. We investigated the FRET between pendrin (by engineering the enhanced cyano fluorescent protein (ECFP) to the $C$ terminus of pendrin, PDS-ECFP) and the plasma membrane (using a construct containing the enhanced yellow fluorescent protein (EYFP) with an N-terminal sequence tag that is post-translationally palmitoylated and targeted to the plasma membrane, EYFP-Mem) in both fixed and living bronchial epithelial cells (NCI-H292). Our studies revealed that forskolin stimulated increases in intracellular cAMP caused a significant increase of pendrin abundance at the plasma membrane. Importantly, the increased pendrin trafficking to the cell surface was associated with a significant increase in $\mathrm{Cl} / \mathrm{HCO}_{3}$ exchange, which lead to intracellular acidification. Moreover, we found 
that insertion of pendrin into the plasma membrane was likely facilitated by a forskolinstimulated depolymerization of actin filaments via Rho-GTPases.

\section{Materials and Methods}

\section{Chemicals and reagents}

All chemicals were purchased from Sigma (Sigma-Aldrich, Milan, Italy). Forskolin was from Fermentek Biotechnology. 2',7'-bis(carboxyethyl)-5(6)-carboxyfluorescein acetoxymethyl ester (BCECF-AM), media and sera for cell culture were from Life Technologies (Life Technologies, Monza, Italy).

\section{Plasmids}

The open reading frame (ORF) of human pendrin from normal thyroid tissue was subcloned by PCR from the pTARGET (Promega Corporation) vector (originally provided by Prof. P. Beck-Peccoz, University of Milan, Italy) into the XhoI and BamHI restriction sites of the pECFPN1 (Clontech) vector. When transfected into mammalian cells, this construct leads to the production of pendrin with a C-terminal ECFP tag.

The CFP(d)Del-EPAC(dDEPCD)-cp173Venus(d)-Venus(d) (Exchange Protein activated by cAMP (EPAC) probe) used to monitor the intracellular concentration of cAMP, was obtained from Jalink Kees [19].

The Raichu-Rho-binding domain (RBD) construct was previously described by Yoshizaki et al. [20], and kindly provided by Prof. Matsuda, (Osaka University, Japan).

The pEYFP-Mem construct was from Clontech. Transfection of cells with this construct results in a strong labeling of the plasma membrane.

\section{Cell culture and transfection}

NCI-H292 cells (from ATCC) were grown in Advanced RPMI 1640 Media (Life Technologies, Monza, Italy) supplemented with $10 \%$ (v/v) fetal bovine serum and $100 \mathrm{i} . \mathrm{u} . / \mathrm{ml}$ penicillin, $100 \mu \mathrm{g} / \mathrm{ml}$ streptomycin at $37^{\circ} \mathrm{C}$ with $5 \% \mathrm{CO}_{2}$. $\mathrm{NCI}-\mathrm{H} 292$ cells were seeded on $40 \mathrm{~mm}$ diameter glass coverslips and grown for $24 \mathrm{~h}$ ( $\sim 80 \%$ confluence). For FRET experiments, cells were transiently transfected with plasmids ( $0.4 \mu \mathrm{g}$ of DNA/ $\left.\mathrm{cm}^{2}\right)$ using TransIT®-2020 transfection reagent $\left(0.75 \mu \mathrm{l} / \mathrm{cm}^{2}\right)$ according to the protocol provided by the manufacturer (Mirus, Mirus Bio LLC, USA). FRET experiments were performed 48 hours post-transfection.

\section{Video Imaging Measurements}

For FRET and intracellular pH measurements, the coverslips were mounted in a perfusion chamber (FCS2 Closed Chamber System, BIOPTECHS, Butler, U.S.A.) and measurements were performed using an inverted microscope (Nikon Eclipse TE2000-S) equipped for single-cell fluorescence measurements and imaging analysis. The sample was illuminated through a 40X oil immersion objective (NA $=1.30)$.

\section{FRET measurements}

FRET experiments were performed as previously described [21, 22]. Briefly, NCI-H292 cells were co-transfected (transiently) with PDS-ECFP and EYFP-Mem, with Raichu-RBD to evaluate Rho activity or with the EPAC probe to measure changes in cAMP. Visualization of ECFP- and/or EYFP-expressing cells and detection of FRET was performed on an inverted microscope (Nikon Eclipse TE2000-S) equipped with a monochromator controlled by MetaMorph/MetaFluor software. ECFP was excited at $433 \mathrm{~nm}$ and EYFP at $512 \mathrm{~nm}$. All images were aligned and corrected for background in the emission windows for FRET (535/30 $\mathrm{nm})$, ECFP $(475 / 30 \mathrm{~nm})$ and EYFP $(535 / 26 \mathrm{~nm})$. Each image was further corrected for ECFP cross-talk and EYFP cross-excitation. Thus, netFRET = IFRETbg $-($ ICFPbg $\mathrm{x}$ a) $-($ IYFPbg - b), where IFRETbg, ICFPbg, and IYFPbg are the background-corrected pixel gray values measured in the FRET, ECFP and EYFP windows, respectively; and $\mathrm{a}$ and $\mathrm{b}$ are the relative contribution to the fluorescence intensity in the FRET window by ECFP cross-talk and EYFP cross-excitation, respectively. The obtained netFRET values were normalized for the protein expression levels (NFRET= netFRETx 100/(ICFPbg x IYFPbg) $\left.{ }^{1 / 2}\right)$. The integrated fluorescence density values of the images from ten regions of interest in each cell (near the cell border) were analyzed using MetaMorph and Microsoft Excel software. Because the fluorescence of green fluorescent protein variants is sensitive to $\mathrm{pH}$ [23], FRET could be affected by pendrin-dependent intracellular acidification. 


\section{Intracellular $\mathrm{pH}$ measurements}

NCI-H292 cells were loaded with $1 \mu \mathrm{M}$ BCECF-AM for $30 \mathrm{~min}$ at $37^{\circ} \mathrm{C}$ in Dulbecco's modified Eagle's medium and then washed in Ringer's Solution containing (in mmol/L): $130 \mathrm{NaCl}, 3 \mathrm{KCl}, 0.5 \mathrm{MgCl}_{2}, 1.2$ $\mathrm{NaHCO}_{3}, 10$ glucose, $1.2 \mathrm{CaCl}_{2}, 10$ HEPES, pH 7.4. BCECF was excited at 490 and $440 \mathrm{~nm}$. Emitted fluorescence was passed through a single band dichroic mirror and filtered at 535nm (Omega Optical, Brattleboro, VT, USA). The BCECF fluorescence intensity ratio was calibrated at the end of each experiment with a calibration solution containing $10 \mu \mathrm{M}$ nigericin and high potassium (in mmol/L: $5 \mathrm{NaCl}, 130 \mathrm{KCl}, 0.1 \mathrm{MgCl}_{2}, 10$ glucose, $1.2 \mathrm{CaCl}_{2}, 10 \mathrm{HEPES}$, pH 5.30 - 8.44 adjusted with $\mathrm{KOH}$ or $\left.\mathrm{HCl}\right)$ [24]. $\mathrm{pH}$ variations ( $\left.\Delta \mathrm{upH}\right)$ were calculated with (pHf-pH0)/pH0, where $\mathrm{pHO}$ is the $\mathrm{pH}$ before forskolin stimulation and $\mathrm{pHf}$ is the maximal $\mathrm{pH}$ reached after forskolin stimulation.

\section{Actin staining}

NCI-H292 cells were grown on $12 \mathrm{~mm}$ diameter glass coverslips, left untreated or stimulated with forskolin $(100 \mu \mathrm{M}$ for $45 \mathrm{~min}$ ) and fixed with $4 \%$ paraformaldehyde in phosphate buffered saline (PBS) for 20 minutes. Cells were washed 3 times for 5 minutes in PBS and permeabilized with $0.1 \%$ Triton X-100 in PBS for 5 minutes. The actin cytoskeleton was visualized by incubation with Phalloidin-tetramethylrhodamine isothiocyanate (Phalloidin-TRITC, $100 \mu \mathrm{g} / \mathrm{ml}$ ) for 45 minutes. Coverslips were mounted on glass slides with Mowiol (Sigma-Aldrich, Milan, Italy) mounting medium and analyzed by confocal microscope (Leica TCS SP2, Leica Microsystems, Heerbrugg, Switzerland).

\section{Statistical analysis}

Data are reported as mean values \pm SEM. Statistical analyses were performed by Student's t-test (unpaired or paired when applicable) with $\mathrm{p}<0.05$ considered statistically significant and where $\mathrm{n}$ represents the number of cells.

\section{Results}

Quantification of pendrin abundance at plasma membrane by FRET studies

In order to quantify the abundance of pendrin expressed at the plasma membrane, we applied a method based on the physical process of FRET in fixed and living cells. As stated above, ECFP functioned as the FRET donor and EYFP as the FRET acceptor. NCI-H292 cells were co-transfected with constructs containing pendrin C-terminally tagged with ECFP (PDS-ECFP), and EYFP-Mem (composed of a fusion protein consisting of EYFP N-terminally tagged with 20 amino acids of neuromodulin, which contain a post-translational palmitoylation signal that targets the construct to the plasma membrane [21, 25]). For FRET to occur, the two fusion proteins must be in close proximity to one another, i.e. pendrin is inserted in or located close to the plasma membrane (Fig. 1A) [26]. To evaluate and test the sensitivity of this approach, NFRET was calculated in fixed NCI-H292 cells treated for 45 min with forskolin $(100 \mu \mathrm{M})$, an activator of cAMP/PKA signaling that is known to regulate the subcellular localization and function of pendrin [13-15, 17], or the vehicle (control). Compared to untreated cells, the FRET signal was significantly increased in cells treated with forskolin $(100 \pm 2.73, \mathrm{n}=104$ and $* 129.1 \pm 5.32, \mathrm{n}=123$ for control and forskolin treated cells, respectively, where $* p<0.0001$ ) (Fig.1B). This finding is consistent with an increase of pendrin abundance at the plasma membrane upon activation of the cAMP/PKA signal transduction pathway.

The dynamic trafficking of pendrin to the plasma membrane was further investigated in living cells. Variations in fluorescence intensity in the FRET channel (535/475nm Emission Ratio) emitted upon excitation at 433nm (Fig. 1C) were evaluated by singlecell epifluorescence imaging and quantified with respect to the levels detected before forskolin stimulation measuring the maximal change in (F1-F0)/F0, where F0 is the average fluorescence intensity in the FRET channel before forskolin stimulation and F1 is the average fluorescence intensity in the FRET channel after forskolin stimulation for $5 \mathrm{~min}$. (F1-F0)/ 
Fig. 1. A, schematic model of FRET between the PDS-ECFP fusion protein (donor) and EYFP-Mem (acceptor). ECFP is fused to the C-terminus of pendrin (PDS) and EYFP is attached to the cell membrane by its lipid anchor. FRET occurs only if the two proteins are within a distance of 1-10 $\mathrm{nm}$. B, representative cell showing the FRET signal under control (CTR) and forskolin stimulation $(\mathrm{FK}, 100 \mu \mathrm{M}$ for $45 \mathrm{~min}$ ). Histograms (means \pm SEM) represent changes in NFRET between control (CTR) and forskolin (FK) treated cells. C, dynamic FRET experiments. Fluorescence intensity in the FRET channel emitted upon excitation at $433 \mathrm{~nm}$. Real time fluorescence intensity was recorded and the (F1-F0)/F0 (see results) was calculated. Original tracings are representative of PDSoverexpressing and mock cells.

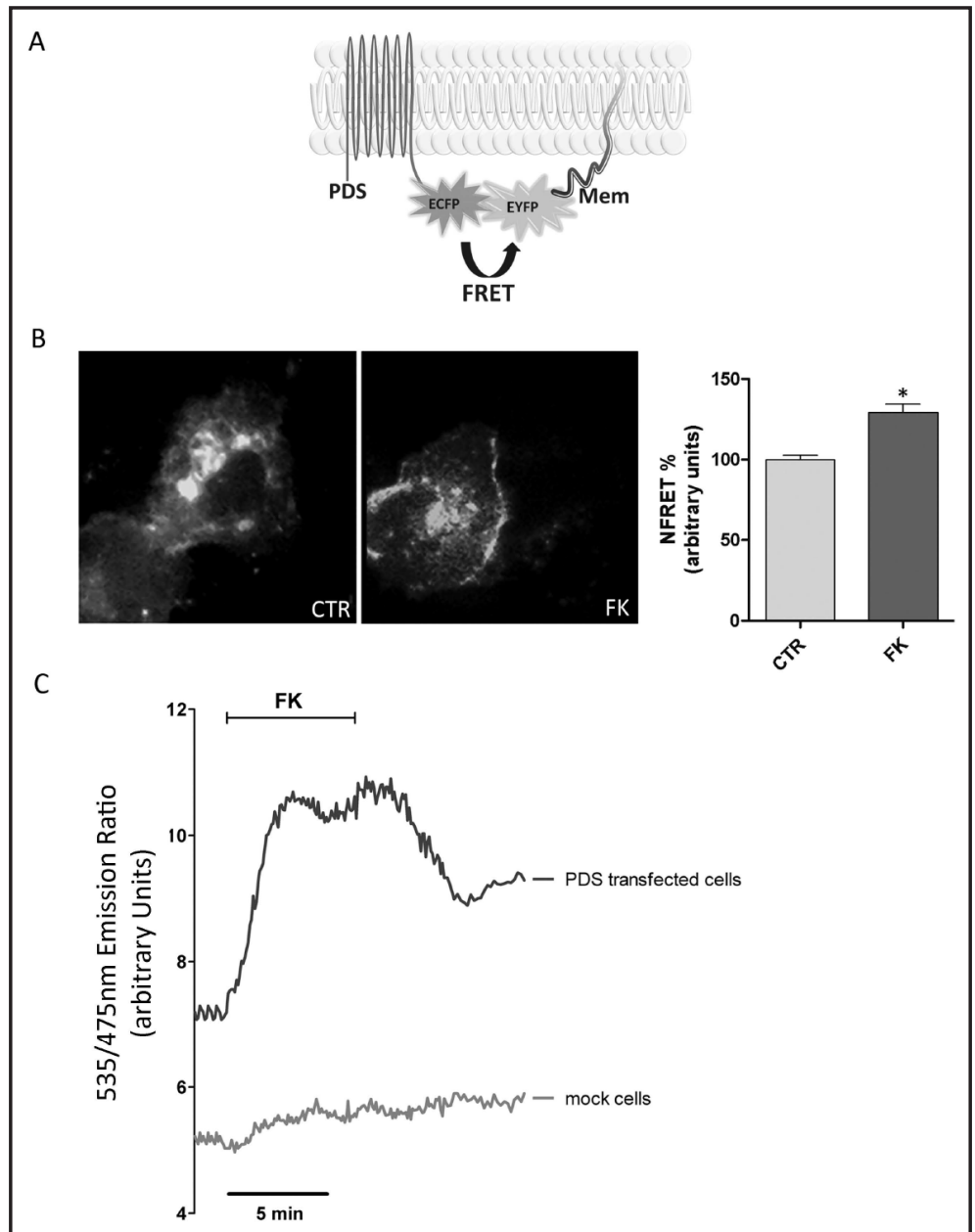

Fig. 2. Representative experimental tracings showing the effect of forskolin stimulation $(\mathrm{FK}, 100 \mu \mathrm{M}$ for $5 \mathrm{~min}$ ) on intracellular $\mathrm{pH}$ in pendrin overexpressing and mock cells. In the inset, histograms (means \pm SEM) represent the intracellular $\mathrm{pH}$ variation $(\Delta \mathrm{upH})$ following forskolin stimulation $\left({ }^{*} \mathrm{p}<0.0001\right)$.

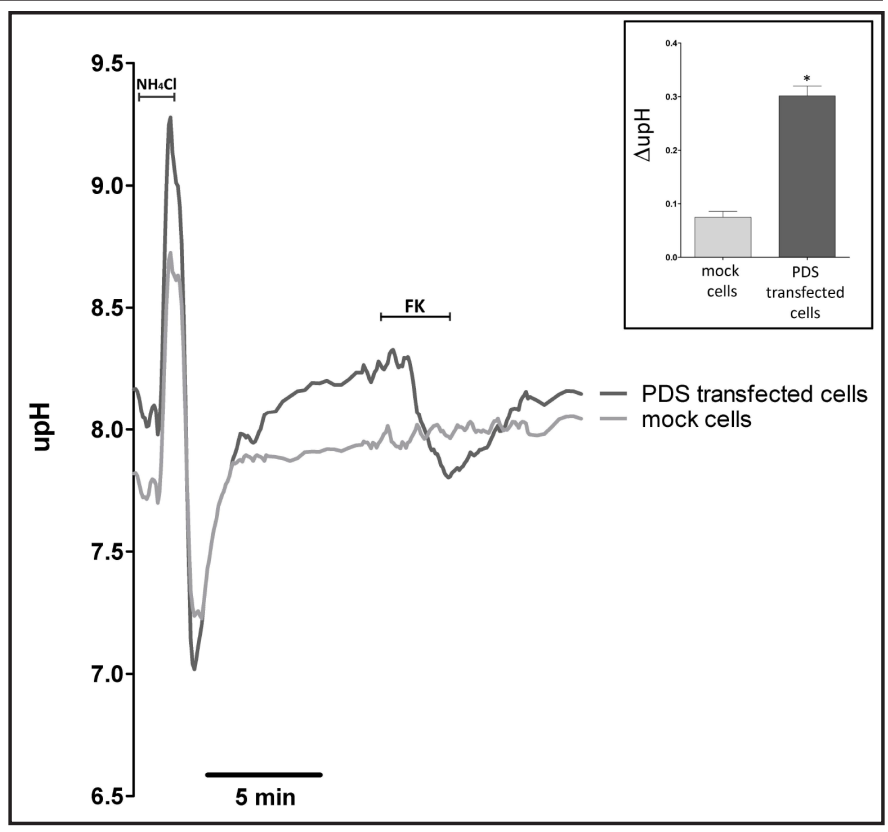

F0 increased by $27.50 \% \pm 1.5(n=56, p<0.0001)$ in pendrin expressing cells. No significant variation in (F1-F0)/F0 was detected in cells transfected with EYFP-Mem and ECFP alone (mock cells). 


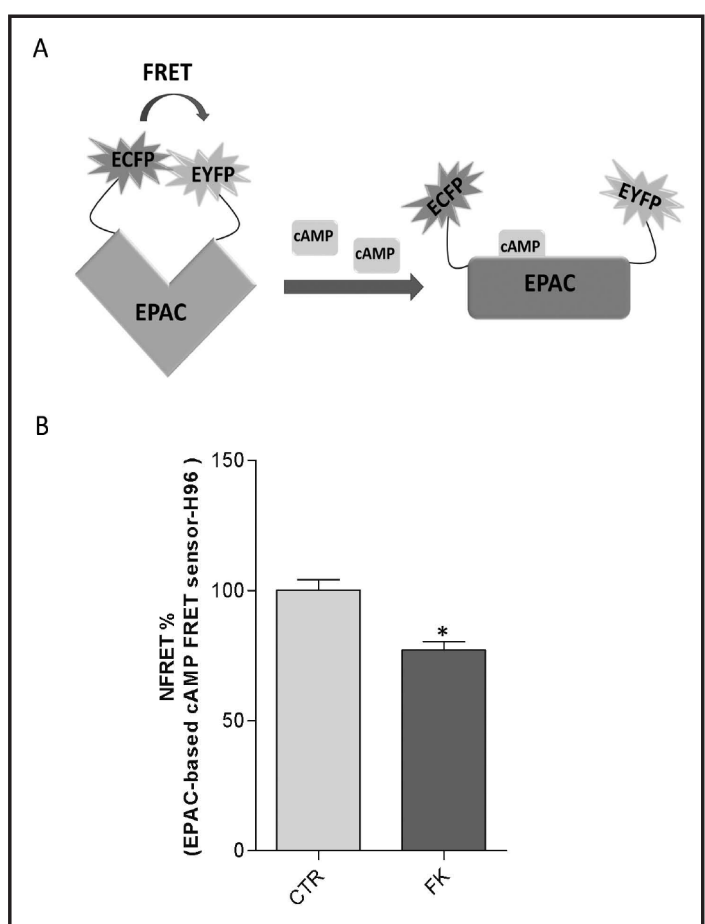

Fig. 3. A, schematic model of a FRET probe containing the cAMP binding sequence of EPAC sandwiched between ECFP (donor) and EYFP (acceptor). Binding of cAMP to EPAC results in an intramolecular steric conformational change causing a significant increase in the distance between the fluorescent donor and acceptor, thereby decreasing FRET. B, histograms (means \pm SEM) represent changes in NFRET between control (CTR) and forskolin (FK, $100 \mu \mathrm{M}$ for $45 \mathrm{~min}$ ) treated cells $\left({ }^{*} \mathrm{p}<0.0001\right)$.
A

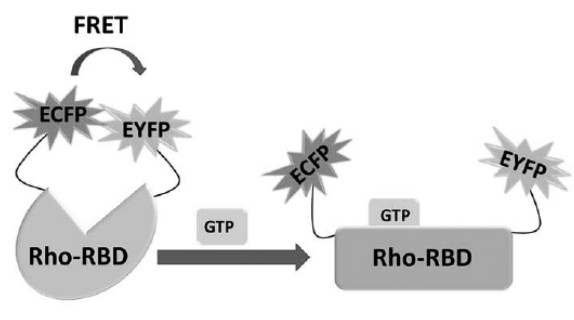

B

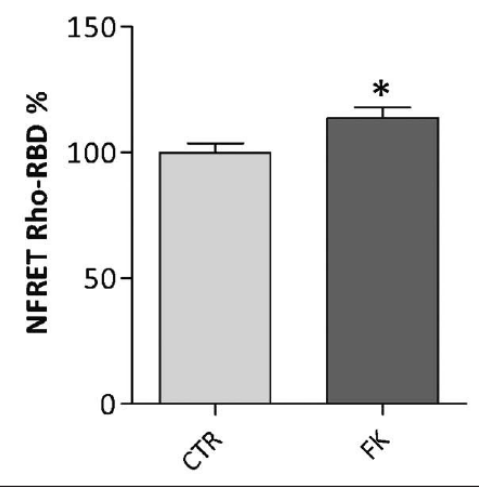

Fig. 4.A, schematic model ofa FRET sensor containing the Rho-binding domain (RBD) sandwiched between ECFP (donor) and EYFP (acceptor). Binding of GTP to the RBD results in an intramolecular conformational change causing a significant increase in the distance between the fluorescent donor and the acceptor, thereby decreasing FRET. B, histograms (means \pm SEM) represent changes in NFRET between control (CTR) and forskolin (FK, $100 \mu \mathrm{M}$ for $45 \mathrm{~min}$ ) treated cells $(* p<0.05)$.

\section{Evaluation of pendrin activity by real-time measurement of intracellular $\mathrm{pH}$}

In most $\mathrm{HCO}_{3}^{-}$secreting epithelial cells, pendrin plays a role in modulating the $\mathrm{pH}$ of secreted fluid [17]. $\mathrm{HCO}_{3}{ }^{-}$secretion via pendrin is accompanied by intracellular acidification [27]. To evaluate whether the increased abundance of pendrin at the plasma membrane upon cAMP/PKA activation is paralleled by an increase in its activity, real-time measurements of intracellular $\mathrm{pH}$ were performed. To this end, NCI-H292 cells transfected with PDS-ECFP were loaded with the pH-sensitive dye BCECF-AM $(1 \mu \mathrm{M}$ for $30 \mathrm{~min})$. Forskolin stimulation resulted in a significant intracellular acidification in pendrin over-expressing cells, whereas no relevant variation in intracellular $\mathrm{pH}$ was detected in not transfected cells (mock cells) $(\Delta \mathrm{upH}=0.074 \pm 0.011, \mathrm{n}=43$ and $* 0.301 \pm 0.018, \mathrm{n}=35$ for mock and PDS -transfected cells, respectively, where ${ }^{*} \mathrm{p}<0.0001$ ) (Fig. 2). These observations are consistent with an increase in pendrin function following activation of cAMP/PKA signaling.

\section{Involvement of Rho proteins as downstream effectors of the cAMP/PKA signal transduction} pathway

Actin remodeling is fundamental in controlling the trafficking and cellular distribution of different channels and transporters $[28,29]$. To further investigate the molecular machinery controlling the cellular localization of pendrin following forskolin stimulation, we evaluated: 1) cAMP concentrations, 2) Rho activity and 3) actin cytoskeleton reorganization. NCI-H292 cells were transfected with a FRET sensor incorporating the cAMP binding sequence of EPAC 
Fig. 5. Effect of forskolin stimulation on actin reorganization in NCI-H292 cells. Cells were treated with vehicle (CTR) or stimulated with forskolin (FK, $100 \mu \mathrm{M}$ for 45 min). F-actin was stained with phalloidin-TRITC and visualized by confocal microscopy.
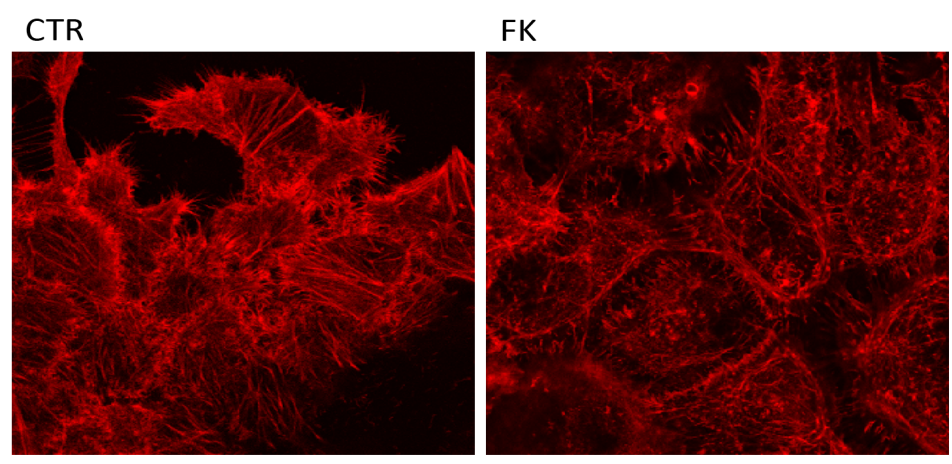

sandwiched between ECFP and EYFP [30]. Binding of cAMP to EPAC causes an intramolecular conformational change resulting in an increase in the distance between the FRET donor and acceptor, thereby decreasing the FRET signal (Fig. 3A). Compared with the vehicle-treated cells (CTR), stimulation with forskolin decreased the calculated NFRET values $(100 \pm 4.2$, $\mathrm{n}=54$ and ${ }^{*} 77 \pm 3.46, \mathrm{n}=51$ for CTR and forskolin treated cells, respectively, where $* \mathrm{p}<$ 0.0001 ), consistent with a significant increase in the intracellular cAMP concentration and consequent activation of PKA (Fig. 3B).

RhoA GTPase is a known target of PKA. Phosphorylation of RhoA GTPase at Ser188 inhibits its activity by increasing the binding between it and its inhibitor, Rho-GDI [31,32]. In order to verify if the forskolin-induced increase in intracellular cAMP was paralleled by decreased Rho activity in our experimental system, NCI-H292 cells were transfected with a FRET sensor (Raichu-RBD) containing the Rho-binding-domain (RBD) sandwiched between ECFP and EYFP [20]. Binding of endogenous GTP-RhoA to the RBD displaces EYFP and ECFP, thereby decreasing FRET efficiency (Fig. 4A). The results of the FRET experiments are summarized in Fig. 4B. Compared to the vehicle-treated cells (CTR), forskolin stimulation significantly increased the NFRET signal, consistent with a decrease in RhoA activity (100 $\pm 3.64, \mathrm{n}=102$ and ${ }^{*} 113.8 \pm 4.16, \mathrm{n}=109$ for CTR and forskolin treated cells, respectively, where ${ }^{*} \mathrm{p}<0.05$ ) (Fig. 4B). Rho inhibition is indeed associated with actin depolymerization [32-34]. Confocal imaging studies confirmed that stimulation of NCI-H292 cells with forskolin caused a significant decrease in actin filaments, consistent with a reorganization of the actin cytoskeleton (Fig. 5).

\section{Discussion}

In this report, we described a FRET-based approach for evaluating the abundance of pendrin at the plasma membrane in cultured bronchial epithelial (NCI-H292) cells stimulated with forskolin. Recent studies have demonstrated that not only loss of function, but also upregulation of pendrin activity might play a key role in the onset of several human disorders [5], underlying the necessity for reliable tools for quantitative detection of membraneexpressed pendrin under different patho/physiological conditions. FRET technology was first described as a powerful tool for studying protein-protein interactions in living cells [35]. Later, it became evident that FRET could also be applied for measuring a wide range of cellular responses such as changes in intracellular calcium and metal concentrations, $\mathrm{pH}$, voltage, enzyme activity and local changes in the concentration of second messengers [36, 37].

Here, using an EPAC-based cAMP FRET sensor, we confirmed that forskolin, a known adenylate cyclase activator, caused a significant decrease in NFRET, which is consistent with an increase in intracellular cAMP levels in NCI-H292 cells (Fig. 3). To evaluate specific and discrete alterations of pendrin abundance at the cell surface, we took advantage of a 
FRET acceptor genetically engineered to target to the plasma membrane (EYFP-Mem). This specific fluorescent sensor provides a quantitative indication of the distribution of membrane proteins with high spatiotemporal resolution. We demonstrated that forskolin significantly increased the FRET signal in both fixed (Fig. 1B) and living (Fig. 1C) NCI-H292 cells co-expressing EYFP-Mem and PDS-ECFP (Fig. 1A), consistent with an increase in pendrin expression at the plasma membrane. In line with a role for $\mathrm{HCO}_{3}^{-}$secretion in airway epithelial tissue [17], the increase in pendrin abundance that we detected at the cell membrane was paralleled by a significant intracellular acidification (Fig. 2). Similarly to that shown in thyroid and kidney $[14,15]$, our data indicate that stimulation of the cAMP signal transduction pathway also increases the amount of pendrin expressed at the cell surface and its transport activity in cultured bronchial NCI-H292 cells. Pendrin transposition to the cell membrane is likely regulated by PKA, since mutation of the putative PKA phosphorylation site (T717A) resulted in a diminished translocation of the transporter to the plasma membrane in response to forskolin in thyroid cells [14]. Further studies will be performed to clarify if PKA phosphorylation plays a role in regulating pendrin localization and function in bronchial epithelial cells. To provide further insight into the molecular signals controlling the cellular distribution of pendrin, we focused on Rho-GTPases, which are specific downstream targets of the cAMP/PKA cascade, and modulate intracellular trafficking events [32, 38-40]. The Rho family of proteins (Rho, Rac, and $\mathrm{Cdc} 42$ ) are active in their GTP-bound state and inactive in their GDP-bound state [38]. We have previously demonstrated that forskolin stimulation resulted in a significant increase in RhoA phosphorylation at S188, a posttranslational modification that stabilizes the inactive form of RhoA. Following phosphorylation of RhoA by PKA, Rho can indeed uncouple from its downstream effectors such as Rho-kinase and the ERM proteins [28, 32, 41, 42]. Using a specific FRET probe generated for measuring Rho activity [20], we showed that forskolin stimulation is associated with a significant decrease in Rho activity (Fig. 4). The attenuation of Rho activity was shown to promote a partial depolymerization of the actin cytoskeleton, which might be considered a pivotal prerequisite facilitating the intracellular trafficking of proteins [32, 43, 44]. Accordingly, stimulation of NCI-H292 cells with forskolin led to a significant reorganization of actin cytoskeleton (Fig. 5). To our knowledge, this is the first report showing the involvement of Rho signaling in NCI-H292 cells under cAMP/PKA stimulation. Whether or not Rho proteins directly or indirectly affect the cellular distribution of pendrin via actin depolymerization remains to be further investigated.

To conclude, herein we propose a FRET-based approach for quantitative detection of pendrin expression at the plasma membrane in fixed and living NCI-H292 cells. This fluorescence assay has a high spatiotemporal resolution, is accurate, is flexible in terms of incubation time and could be applied in several cell models to monitor even minute changes of pendrin expression at the plasma membrane in real time.

\section{Conflict of Interest}

The authors have no conflict of interest to declare.

\section{Acknowledgements}

This study was funded by grants from University of Bari, Italy (Idea Giovani 2011), and PRIN (Research Program of National Interest) projects to G. Tamma (Tamma01373409Prin). C. Nofziger is supported by the Roche Postdoc Fellowship Program (\#231). This work was further supported by the FWF (P18608) and the FP-7 (PIRSES-GA-2008-230661) grants to M. Paulmichl. 


\section{Cellular Physiology and Biochemistry}

Cell Physiol Biochem 2013;32(suppl 1):200-209

\begin{tabular}{l|l} 
DOI: $10.1159 / 000356639$ & C 2013 S. Karger AG, Basel
\end{tabular}

www.karger.com/cpb

Tamma et al.: Forskolin-Induced Pendrin Trafficking at the Plasma Membrane

\section{References}

1 Kohn LD, Suzuki K, Nakazato M, Royaux I, Green ED: Effects of thyroglobulin and pendrin on iodide flux through the thyrocyte. Trends Endocrinol Metab 2001;12:10-16.

-2 Pedemonte N, Caci E, Sondo E, Caputo A, Rhoden K, Pfeffer U, Di Candia M, Bandettini R, Ravazzolo R, Zegarra-Moran O, Galietta LJ: Thiocyanate transport in resting and IL-4-stimulated human bronchial epithelial cells: Role of pendrin and anion channels. J Immunol 2007;178:5144-5153.

-3 Soleimani M: Molecular physiology of the renal chloride-formate exchanger. Curr Opin Nephrol Hypertens 2001;10:677-683.

-4 Bronckers AL, Guo J, Zandieh-Doulabi B, Bervoets TJ, Lyaruu DM, Li X, Wangemann P, DenBesten P: Developmental expression of solute carrier family 26a member 4 (slc26a4/pendrin) during amelogenesis in developing rodent teeth. Eur J Oral Sci 2011;119:S185-192.

5 Dossena S, Nofziger C, Lang F, Valenti G, Paulmichl M: The ESF meeting on "The proteomics, epigenetics and pharmacogenetics of pendrin". Cell Physiol Biochem 2011;28:377-384.

6 Fugazzola L, Cerutti N, Mannavola D, Vannucchi G, Beck-Peccoz P: The role of pendrin in iodide regulation. Exp Clin Endocrinol Diabetes 2001;109:18-22.

7 Soleimani M, Greeley T, Petrovic S, Wang Z, Amlal H, Kopp P, Burnham CE: Pendrin: An apical Cl/ $/ \mathrm{OH}^{-} / \mathrm{HCO}_{3}$ exchanger in the kidney cortex. Am J Physiol Renal Physiol 2001;280:F356-364.

Bizhanova A, Kopp P: Genetics and phenomics of pendred syndrome. Mol Cell Endocrinol 2010;322:83-90. Everett LA, Glaser B, Beck JC, Idol JR, Buchs A, Heyman M, Adawi F, Hazani E, Nassir E, Baxevanis AD, Sheffield VC, Green ED: Pendred syndrome is caused by mutations in a putative sulphate transporter gene (pds). Nat Genet 1997;17:411-422.

10 Glaser B: Pendred syndrome. Pediatr Endocrinol Rev 2003;1 Suppl 2:199-204; discussion 204.

-11 Scott DA, Wang R, Kreman TM, Sheffield VC, Karniski LP: The pendred syndrome gene encodes a chlorideiodide transport protein. Nat Genet 1999;21:440-443.

>12 Nofziger C, Vezzoli V, Dossena S, Schonherr T, Studnicka J, Nofziger J, Vanoni S, Stephan S, Silva ME, Meyer G, Paulmichl M: Stat6 links IL-4/IL-13 stimulation with pendrin expression in asthma and chronic obstructive pulmonary disease. Clin Pharmacol Ther 2011;90:399-405.

13 Bizhanova A, Chew TL, Khuon S, Kopp P: Analysis of cellular localization and function of carboxy-terminal mutants of pendrin. Cell Physiol Biochem 2011;28:423-434.

14 Pesce L, Bizhanova A, Caraballo JC, Westphal W, Butti ML, Comellas A, Kopp P: TSH regulates pendrin membrane abundance and enhances iodide efflux in thyroid cells. Endocrinology 2011;153:512-521.

15 Azroyan A, Morla L, Crambert G, Laghmani K, Ramakrishnan S, Edwards A, Doucet A: Regulation of pendrin by cAMP: Possible involvement in beta-adrenergic-dependent $\mathrm{NaCl}$ retention. Am J Physiol Renal Physiol 2012;302:F1180-1187.

16 Thumova M, Pech V, Froehlich O, Agazatian D, Wang X, Verlander JW, Kim YH, Wall SM: Pendrin protein abundance in the kidney is regulated by nitric oxide and cAMP. Am J Physiol Renal Physiol 2012;303:F812820.

17 Garnett JP, Hickman E, Burrows R, Hegyi P, Tiszlavicz L, Cuthbert AW, Fong P, Gray MA: Novel role for pendrin in orchestrating bicarbonate secretion in cystic fibrosis transmembrane conductance regulator (CFTR)-expressing airway serous cells. J Biol Chem 2011;286:41069-41082.

18 Jares-Erijman EA, Jovin TM: FRET imaging. Nat Biotechnol 2003;21:1387-1395.

19 Klarenbeek JB, Goedhart J, Hink MA, Gadella TW, Jalink K: A mTurquoise-based cAMP sensor for both flim and ratiometric read-out has improved dynamic range. PLoS One 2011;6:e19170.

20 Yoshizaki H, Ohba Y, Kurokawa K, Itoh RE, Nakamura T, Mochizuki N, Nagashima K, Matsuda M: Activity of Rho-family GTPases during cell division as visualized with FRET-based probes. J Cell Biol 2003;162:223232.

21 Ritter M, Ravasio A, Jakab M, Chwatal S, Furst J, Laich A, Gschwentner M, Signorelli S, Burtscher C, Eichmuller S, Paulmichl M: Cell swelling stimulates cytosol to membrane transposition of ICln. J Biol Chem 2003;278:50163-50174.

22 Tamma G, Lasorsa D, Ranieri M, Mastrofrancesco L, Valenti G, Svelto M: Integrin signaling modulates AQP2 trafficking via arg-gly-asp (RGD) motif. Cell Physiol Biochem 2011;27:739-748.

-23 Llopis J, McCaffery JM, Miyawaki A, Farquhar MG, Tsien RY: Measurement of cytosolic, mitochondrial, and Golgi pH in single living cells with green fluorescent proteins. Proc Natl Acad Sci U S A 1998;95:6803-6808. 
24 Ozkan P, Mutharasan R: A rapid method for measuring intracellular pH using BCECF-AM. Biochim Biophys Acta 2002;1572:143-148.

25 Ormo M, Cubitt AB, Kallio K, Gross LA, Tsien RY, Remington SJ: Crystal structure of the aequorea victoria green fluorescent protein. Science 1996;273:1392-1395.

26 Sharma AK, Rigby AC, Alper SL: STAS domain structure and function. Cell Physiol Biochem 2011;28:407422.

-27 Dossena S, Bizhanova A, Nofziger C, Bernardinelli E, Ramsauer J, Kopp P, Paulmichl M: Identification of allelic variants of pendrin (SLC26A4) with loss and gain of function. Cell Physiol Biochem 2011;28:467476.

28 Tamma G, Klussmann E, Oehlke J, Krause E, Rosenthal W, Svelto M, Valenti G: Actin remodeling requires ERM function to facilitate AQP2 apical targeting. J Cell Sci 2005;118:3623-3630.

29 Tamma G, Procino G, Strafino A, Bononi E, Meyer G, Paulmichl M, Formoso V, Svelto M, Valenti G: Hypotonicity induces aquaporin-2 internalization and cytosol-to-membrane translocation of ICln in renal cells. Endocrinology 2007;148:1118-1130.

-30 Bos JL: Epac: A new cAMP target and new avenues in cAMP research. Nat Rev Mol Cell Biol 2003;4:733738.

-31 Forget MA, Desrosiers RR, Gingras D, Beliveau R: Phosphorylation states of Cdc42 and RhoA regulate their interactions with Rho GDP dissociation inhibitor and their extraction from biological membranes. Biochem J 2002;361:243-254.

-32 Tamma G, Klussmann E, Procino G, Svelto M, Rosenthal W, Valenti G: cAMP-induced AQP2 translocation is associated with RhoA inhibition through RhoA phosphorylation and interaction with RhoGDI. J Cell Sci 2003;116:1519-1525.

-33 Klussmann E, Tamma G, Lorenz D, Wiesner B, Maric K, Hofmann F, Aktories K, Valenti G, Rosenthal W: An inhibitory role of Rho in the vasopressin-mediated translocation of aquaporin-2 into cell membranes of renal principal cells. J Biol Chem 2001;276:20451-20457.

34 Tamma G, Klussmann E, Maric K, Aktories K, Svelto M, Rosenthal W, Valenti G: Rho inhibits cAMPinduced translocation of aquaporin-2 into the apical membrane of renal cells. Am J Physiol Renal Physiol 2001;281:F1092-1101.

35 Sekar RB, Periasamy A: Fluorescence resonance energy transfer (FRET) microscopy imaging of live cell protein localizations. J Cell Biol 2003;160:629-633.

-36 Lissandron V, Terrin A, Collini M, D'Alfonso L, Chirico G, Pantano S, Zaccolo M: Improvement of a FRETbased indicator for cAMP by linker design and stabilization of donor-acceptor interaction. J Mol Biol 2005;354:546-555.

-37 Pollok BA, Heim R: Using GFP in FRET-based applications. Trends Cell Biol 1999;9:57-60.

-38 Ridley AJ: Rho GTPases and actin dynamics in membrane protrusions and vesicle trafficking. Trends Cell Biol 2006;16:522-529.

39 Frantz C, Coppola T, Regazzi R: Involvement of Rho GTPases and their effectors in the secretory process of PC12 cells. Exp Cell Res 2002;273:119-126.

40 Gasman S, Chasserot-Golaz S, Popoff MR, Aunis D, Bader MF: Involvement of Rho GTPases in calciumregulated exocytosis from adrenal chromaffin cells. J Cell Sci 1999;112:4763-4771.

41 Dong JM, Leung T, Manser E, Lim L: cAMP-induced morphological changes are counteracted by the activated RhoA small GTPase and the Rho kinase ROKalpha. J Biol Chem 1998;273:22554-22562.

42 Tamma G, Procino G, Svelto M, Valenti G: Hypotonicity causes actin reorganization and recruitment of the actin-binding ERM protein moesin in membrane protrusions in collecting duct principal cells. Am J Physiol Cell Physiol 2007;292:C1476-1484.

43 Chen W, Pendyala S, Natarajan V, Garcia JG, Jacobson JR: Endothelial cell barrier protection by Simvastatin: GTPase regulation and NADPH oxidase inhibition. Am J Physiol Lung Cell Mol Physiol 2008;295:L575-583.

44 Gao C, Li R, Liu Y, Ma L, Wang S: Rho-kinase-dependent F-actin rearrangement is involved in the release of endothelial microparticles during IFN-alpha-induced endothelial cell apoptosis. J Trauma Acute Care Surg 2012;73:1152-1160. 